\title{
Dynamic bifurcation analysis and mitigation of SSR in SMIB system
}

\author{
Shruthi Ramachandra, R. C. Mala, H. V. Gururaj Rao \\ Department of Electrical and Electronics Engineering, Manipal Institute of Technology, India
}

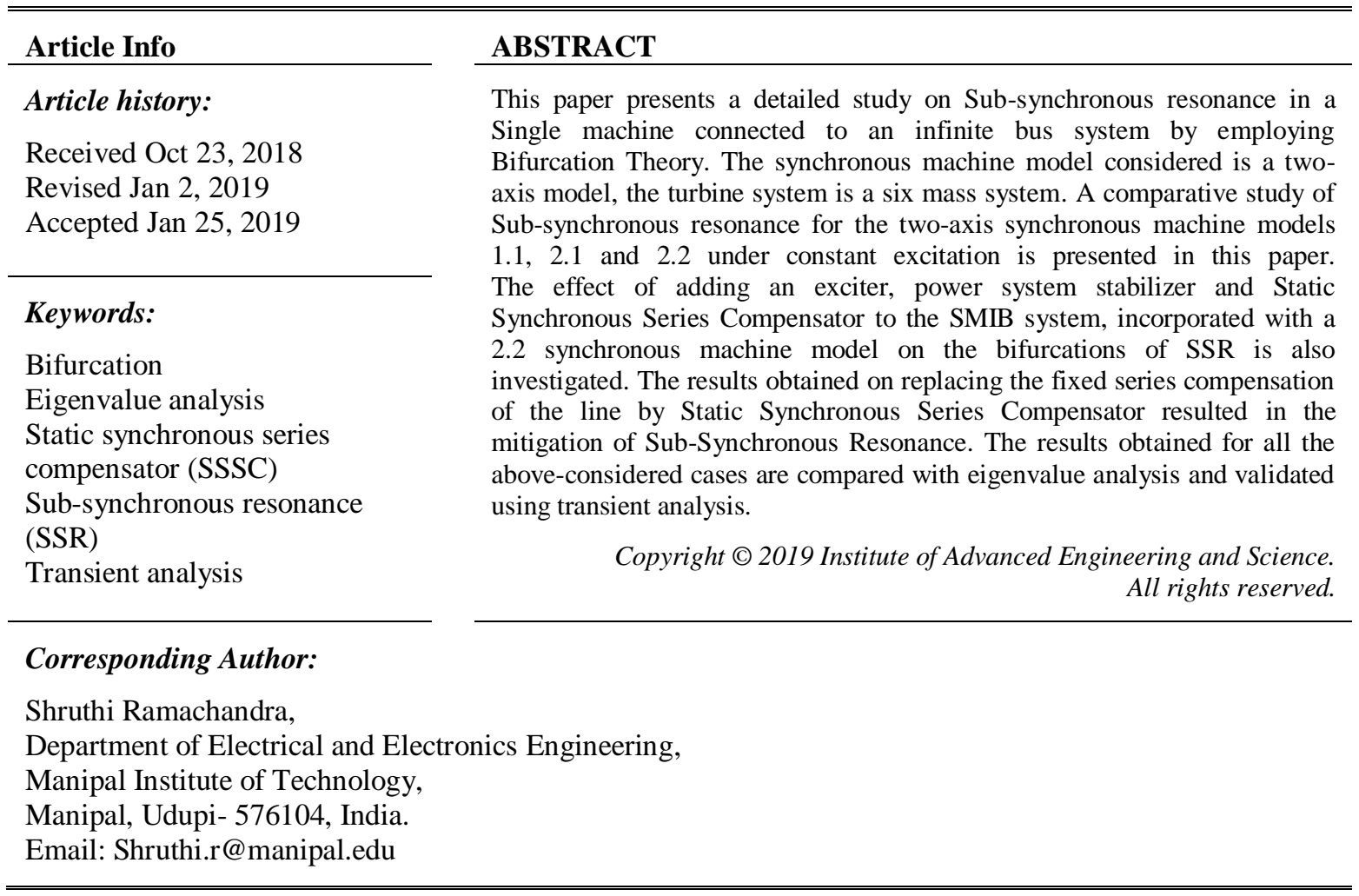

\section{INTRODUCTION}

Series compensation of transmission lines is used to enhance power transfer capability. Series compensation using series connected capacitors increases the maximum power transferred by reducing its effective impedance. The use of series capacitors may, however, lead to sustained oscillations at frequencies lower than the rated frequency. This is broadly known as Sub-Synchronous Resonance [1].

SSR occurs either due to "Induction generator effect" or due to "Torsional Interaction and Amplification Effect". Induction generator effect is caused by self-excitation of the synchronous generators. Torsional Interaction and Amplification Effect occurs due to the interaction of electrical network subsynchronous frequency with the torsional oscillation modes of electro-mechanical components of the power system followed by a disturbance such as variation in electromagnetic torque caused by transients in the network that may be caused due to switching [2].

There are several methods to the study SSR. In this paper, Bifurcation Analysis has been carried out. Transient analysis is used to validate the same. "Bifurcation Theory is used to study possible changes in the structure of the orbits of a differential equation depending on variable parameters" [3]. The value of the parameter at which the system exhibits qualitative change is known as a "bifurcation point". As explained in $[4,5]$ Local bifurcation theory has been applied to provide an explanation for various nonlinear behaviours in power system and power system instabilities.

The problem associated with the application of series capacitors in transmission lines was first discussed in 1937 by J W Butler and C Concordia in [6]. However, the torque shaft oscillations were neglected until the first experience of SSR at the Mohave Generating Station in 1970, followed by another 
identical situation in 1971 [7]. SSR problem in SMIB was studied by Zhu [8] and Zhu et al. [9] using the Hopf bifurcation theorem.

Authors of [10] have implemented phase imbalance scheme to stabilize Sub-synchronous oscillations. FACTS controllers can be used to mitigate SSR [11]. Sub-synchronous resonance in a hybrid compensated system with FACTS controller incorporating energy storage device has been investigated in $[12,13]$.

This paper presents the effect of synchronous machine damper windings on Hopf bifurcations of SSR. It also discusses the impact of static exciter and PSS, on the stability boundary. SSSC with Type-2 controller is used to eliminate Hopf bifurcations of SSR.

\section{SYSTEM DESCRIPTION}

\subsection{SMIB Modelling}

A single machine connected to an infinite bus system is as shown in Figure 1. The voltage at the generator terminal is denoted as $\mathrm{Vg} \angle \theta$. The voltage at the infinite bus is $\mathrm{Eb} \angle 0$. Rt and $\mathrm{Xt}$ are the transformer resistance and reactance respectively. $\mathrm{Rl}$ and $\mathrm{Xl}$ are the resistance and reactance of the line. $\mathrm{Xc}$ is the series compensation.

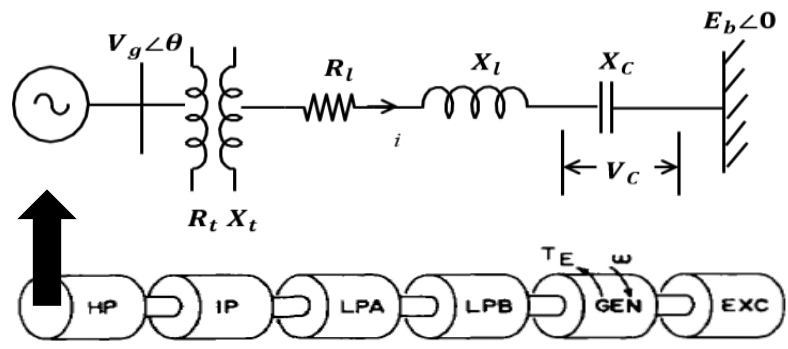

Figure 1. Single machine connected to the infinite bus system

The synchronous machine considered has two damper windings along q-axis. The field winding and one damper winding and along d-axis as described in [14]. As shown in (1) to (9) describe the synchronous machine along the direct and quadrature axis.

d-axis equations:

$$
\begin{aligned}
& \frac{d \Psi_{d}}{d t}=-\omega \Psi_{q}-R_{a} i_{d} \omega_{b}-\omega_{b} V_{d} \\
& \frac{d \Psi_{f}}{d t}=\frac{1}{T_{d}^{\prime}}\left(-\Psi_{F}+\Psi_{d}\right)+\left(\frac{x_{d}^{\prime}}{\left(x_{d}-x_{d}^{\prime}\right)} E_{f d}\right) \\
& \frac{d \Psi_{h}}{d t}=\frac{1}{T_{d}^{\prime \prime}}\left(-\Psi_{h}+\Psi_{d}\right) \\
& \Psi_{d}=x_{d}^{\prime \prime} i_{d}+\left(\frac{x_{d}^{\prime}-x_{d}^{\prime \prime}}{x_{d}^{\prime}}\right) \Psi_{h}+\left(\frac{x_{d}-x_{d}^{\prime}}{x_{d}}\right)\left(\frac{x_{d}^{\prime \prime}}{x_{d}^{\prime}}\right) \Psi_{f}
\end{aligned}
$$

q-axis equations:

$$
\begin{aligned}
& \frac{d \Psi_{q}}{d t}=\omega \Psi_{d}-R_{a} i_{q} \omega_{b}-\omega_{b} V_{q} \\
& \frac{d \Psi_{g}}{d t}=\frac{1}{T_{q}^{\prime}}\left(-\Psi_{g}+\Psi_{q}\right) \\
& \frac{d \Psi_{k}}{d t}=\frac{1}{T_{q}^{\prime \prime}}\left(-\Psi_{k}+\Psi_{q}\right) \\
& \Psi_{q}=x_{q}^{\prime \prime} i_{q}+\left(\frac{x_{q}^{\prime}-x_{q}^{\prime \prime}}{x_{q}^{\prime}}\right) \Psi_{k}+\left(\frac{x_{q}-x_{q}^{\prime}}{x_{q}}\right)\left(\frac{x_{q}^{\prime \prime}}{x_{q}^{\prime}}\right) \Psi_{g}
\end{aligned}
$$


The mathematical expression describing the transmission line in the $\mathrm{d}-\mathrm{q}$ frame is:

$$
\frac{d}{d t}\left[\begin{array}{l}
i_{d} \\
i_{q}
\end{array}\right]=\left[\begin{array}{cc}
\frac{-R \omega_{b}}{X_{e}} & -\omega \\
\omega & \frac{-R \omega_{b}}{X_{e}}
\end{array}\right]\left[\begin{array}{l}
i_{d} \\
i_{q}
\end{array}\right]+\frac{\omega_{b}}{X_{e}}\left(\left[\begin{array}{l}
V_{d} \\
V_{q}
\end{array}\right]-\left[\begin{array}{c}
E_{d} \\
E_{q}
\end{array}\right]-\left[\begin{array}{l}
V_{C d} \\
V_{C q}
\end{array}\right]\right)
$$

The turbine shaft system is modelled as six mass system. The mechanical equations for a single mechanical mass are as follows:

$$
\begin{gathered}
2 H_{i}\left(\frac{d s_{i}}{d t}\right)=\left[T_{m i}-T_{e i}-D_{i}\left(s_{i}-s_{i 0}\right)-D_{i, i-1}\left(s_{i}-s_{i-1}\right)+T_{i, i-1}-D_{i, i+1}\left(s_{i}-s_{i+1}\right)-T_{i, i+1}\right] \\
\frac{d T_{i, i+1}}{d t}=\omega_{b} K_{i, i+1}\left(s_{i}-s_{i+1}\right) \\
\frac{d T_{i, i-1}}{d t}=\omega_{b} K_{i, i-1}\left(s_{i-1}-s_{i}\right) \\
s_{i}=\frac{\omega_{i}-\omega_{b}}{\omega_{b}} \\
\frac{d \delta}{d t}=\omega_{b}\left(s_{g}-s_{g 0}\right)
\end{gathered}
$$

In general, a rotor with ' $p$ ' masses has ' $p-1$ ' torsional modes and one common mode motion i.e., 'Mode 0 '. The $\mathrm{j}^{\text {th }}$ torsional mode has ' $\mathrm{j}$ ' number of polarity reversals in its mode shape [15].

The fast-acting static excitation system is modelled as described in the following equations:

$$
\begin{aligned}
& \frac{d V_{r}}{d t}=\frac{1}{T_{a}}\left[-V_{r}+K_{a}\left(V_{\text {ref }}-V_{t}+V_{s}\right)\right] \\
& \frac{V_{t}^{\prime}}{V_{t}}=\frac{1}{1+s T_{m}}
\end{aligned}
$$

Power system stabilizer (PSS) is modelled using a washout filter and a lead-lag compensator as shown in Figure 2.

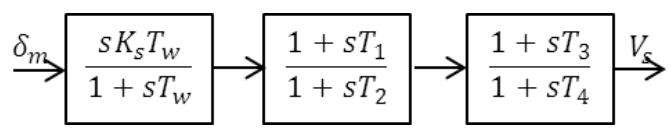

Figure 2. Power system stabilizer

\subsection{Modified SMIB System Equipped with SSSC}

Figure 3 shows the modified SMIB system with the fixed series capacitor replaced by Static Synchronous Series Compensator (SSSC). The equivalent circuit of SSSC is as shown in Figure 4. The operation of SSSC is explained in detail in [16].

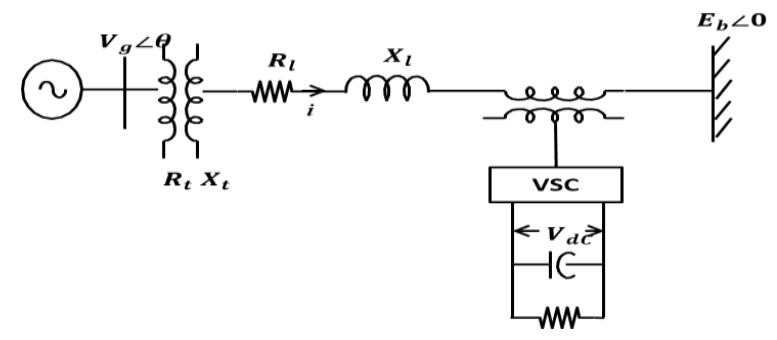

Figure 3. Single machine connected to an infinite bus system equipped with SSSC

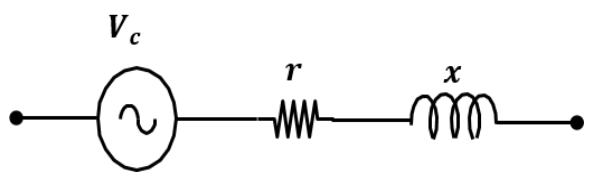

Figure 4. Equivalent circuit of SSSC 


$$
\widehat{V_{C}}=V_{C}(\cos \gamma-j \sin \gamma) e^{j \Phi}=\left(V_{C p}-j V_{C r}\right) e^{j \Phi}
$$

Where $\Phi$ is the phase angle of the line current and $\gamma$ is the angle by which $\mathrm{V}_{\mathrm{C}}$ lags the current. If $\mathrm{V}_{\mathrm{CR}}$ is positive, it implies capacitive mode and $\mathrm{V}_{\mathrm{CR}}$ negative implies inductive mode. Since $\gamma$ is close to $\pm 90^{\circ}$

$$
\gamma=\operatorname{sgn}\left(V_{c r}\right)[-90+\alpha]
$$

The equation for the DC Capacitor voltage is as given below:

$$
\frac{d V_{d c}}{d t}=-\left(\frac{\omega_{b}}{b_{c}}\right) i_{d c}-\left(\frac{g_{c} \omega_{b}}{b_{c}}\right) V_{d c}
$$

Where

$$
i_{d c}=-\left[k_{m} \sin (\Phi+\gamma) i_{d}+k_{m} \cos (\Phi+\gamma) i_{q}\right]
$$

As there is no energy source on the d.c. side, the losses in the converter are neglected. The magnitude of Vc can be controlled to regulate power flow. Type II controller is shown in Figure 5 is used to control the phase angle of the converter voltage in SSSC [16].

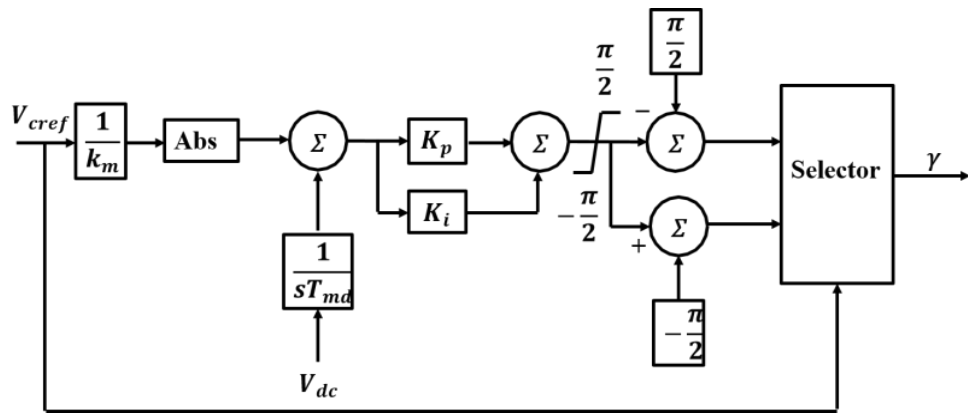

Figure 5. Block diagram of the SSSC Type II controller

\section{RESULTS AND ANALYSIS}

Bifurcation analysis is used to indicate a qualitative change in the dynamics of a system, such as number and type of solutions under the variation of one or more parameters on which the system in question depends. The local stability of an equilibrium solution depends on the eigenvalues of its Jacobian matrix. The equilibrium solution may lose its stability through a static bifurcation or through a Hopf bifurcation.

The conditions for the occurrence of a Hopf point is given in $[4,5]$. If a particular quantity called the first Lyapunov coefficient of the Hopf point is negative, then it is a supercritical bifurcation and the limit cycle is stable. Otherwise, it is a subcritical bifurcation and the limit cycle is unstable.

Six cases are considered for dynamic bifurcation analysis of sub-synchronous resonance. Series compensation is the variable parameter. The study is conducted by varying series compensation from $0 \%$ to $90 \%$ of the line reactance. Matcont $[17,18]$, a dynamic bifurcation toolbox is used to detect the Hopf points. Eigenvalue and transient analysis of the same are carried out using MATLAB-SIMULINK.

\subsection{Effect of Damper Windings}

Case 1: In this case, the synchronous machine is modelled as a 1.1 model, with one damper winding in q-axis and one field winding along d-axis. The SMIB is modelled with constant excitation. Matcont results yielded seven Hopf bifurcation points as shown in Figure 6a. A Limit point is obtained at Xc $=0.083719$, where stable node branch meets the unstable node branch. Figure $6 \mathrm{~b}$ is a plot of real part of eigenvalues corresponding to the five torsional modes under varying Xc. This figure shows that mode 5 and 0 remain stable all along. However, modes 4, 3, 2 and 1 yield seven Hopf points. The value of series capacitance corresponding to these Hopf points, their respective first Lyapunov Coefficients and the nature of these Hopf points are given in Table 1. 


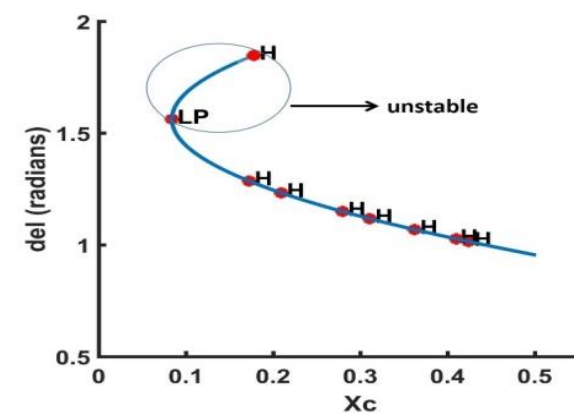

Figure 6a. Dynamic Bifurcations of SSR with 1.1 synchronous machine model

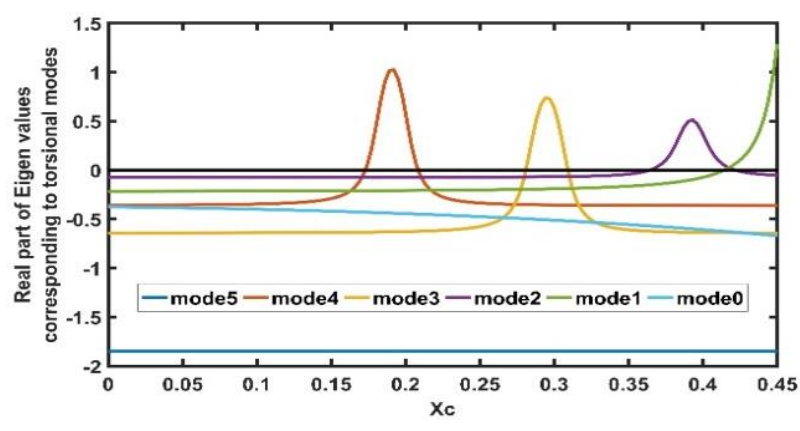

Figure $6 \mathrm{~b}$. Real part of eigenvalues corresponding to torsional modes v/s Xc for Case 1

Table 1. Xc Values Corresponding to Hopf Points and Their First Lyapunov Coefficients for Case 1

\begin{tabular}{ccc}
\hline Xc pu & First Lyapunov Co-effiecient & \\
\hline 0.172157 & $-1.177592 \mathrm{e}-04$ & Supercritical \\
0.209155 & $1.959697 \mathrm{e}-04$ & Subcritical \\
0.279417 & $-2.897224 \mathrm{e}-04$ & Supercritical \\
0.310398 & $6.646034 \mathrm{e}-04$ & Subcritical \\
0.362025 & $-6.948669 \mathrm{e}-05$ & Supercritical \\
0.409705 & $-2.997175 \mathrm{e}-04$ & Supercritical \\
0.423740 & $6.370813 \mathrm{e}-05$ & Subcritical \\
\hline
\end{tabular}

Case 2: In this case, the synchronous machine is modelled as a 2.1 model, with a field winding and a damper winding along $\mathrm{d}$-axis and one damper winding along q-axis. The single machine connected to the Infinite bus is modelled with a constant excitation system. Figure $7 \mathrm{a}$ and Figure $7 \mathrm{~b}$ show the seven Hopf points. A limit point is obtained at Xc $=0.083719$ pu beyond which the system becomes unstable. The value of series capacitance corresponding to these Hopf points, their respective first Lyapunov Coefficients and the nature of these Hopf points are tabulated in Table 2.

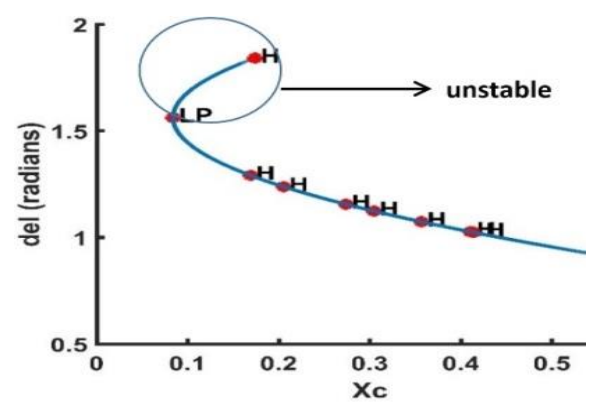

Figure 7a. Dynamic Bifurcations of SSR with 2.1 synchronous machine model

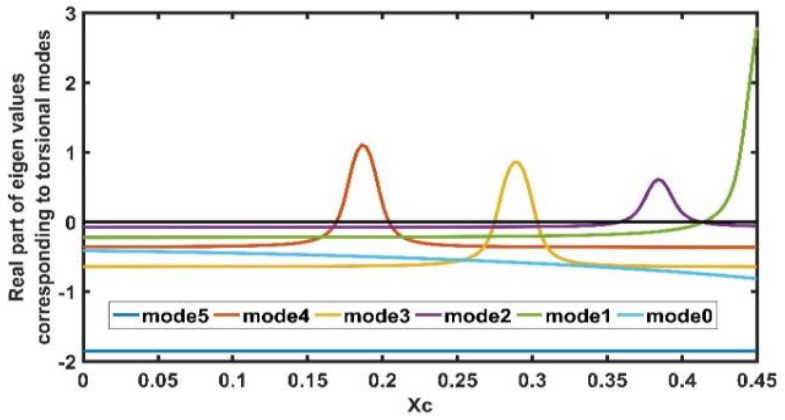

Figure 7b. Real part of eigenvalues corresponding to torsional modes v/s Xc for Case 2

Table 2. Xc Values Corresponding to Hopf Points and Their First Lyapunov Coefficients for Case 2

\begin{tabular}{ccc}
\hline $\mathrm{Xc} \mathrm{pu}$ & First Lyapunov Co-efficient & \\
\hline 0.168608 & $-1.081932 \mathrm{e}-04$ & Supercritical \\
0.204927 & $1.891259 \mathrm{e}-04$ & Subcritical \\
0.273618 & $-2.692822 \mathrm{e}-04$ & Supercritical \\
0.304228 & $6.966456 \mathrm{e}-04$ & Subcritical \\
0.356497 & $-7.002592 \mathrm{e}-05$ & Supercritical \\
0.409792 & $-4.527837 \mathrm{e}-04$ & Supercritical \\
0.413498 & $6.002503 \mathrm{e}-05$ & Subcritical \\
\hline
\end{tabular}


Case 3: In this case, the synchronous machine is modelled as a 2.2 model, with a field winding and a damper winding along d-axis and two damper windings along q-axis. The single machine connected to the infinite bus is modelled with static excitation system. Figure 8a and Figure 8b show seven Hopf points. A limit point is obtained at $\mathrm{Xc}=0.069251$ beyond which the system becomes unstable. The nature of the obtained Hopf points and their corresponding first Lyapunov coefficients are tabulated in Table 3.

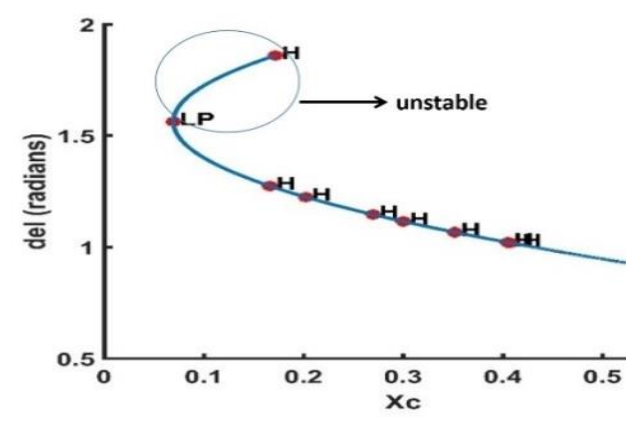

Figure 8a. Dynamic Bifurcations of SSR with 2.2 synchronous machine model

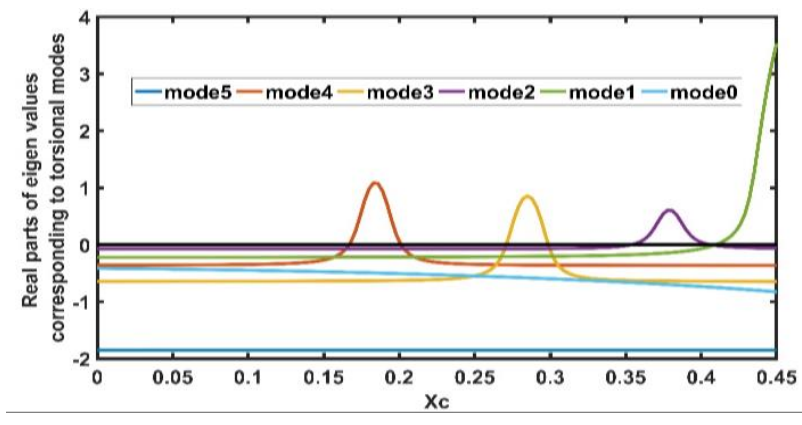

Figure $8 \mathrm{~b}$. Real part of eigenvalues corresponding to torsional modes v/s Xc for Case 3

Table 3. Xc Values Corresponding to Hopf Points and Their First Lyapunov Coefficients for Case 3

\begin{tabular}{ccc}
\hline $\mathrm{Xc} \mathrm{pu}$ & First Lyapunov Co-efficient & \\
\hline 0.166041 & $-1.137315 \mathrm{e}-04$ & Super-critical \\
0.202074 & $1.963731 \mathrm{e}-04$ & Sub-critical \\
0.269662 & $-2.819757 \mathrm{e}-04$ & Super-critical \\
0.299953 & $7.561363 \mathrm{e}-04$ & Sub-critical \\
0.351568 & $-7.280541 \mathrm{e}-05$ & Super-critical \\
0.404753 & $-4.778322 \mathrm{e}-04$ & Super-critical \\
0.407537 & $6.786796 \mathrm{e}-05$ & Sub-critical \\
\hline
\end{tabular}

On comparing Table 1, 2 and 3 we observe that with an increase in damper windings in the synchronous machine Hopf points occur at lesser values of series compensation. At $\mathrm{Xc}=0.174 \mathrm{pu}$, the electrical sub-synchronous mode is close to $4^{\text {th }}$ torsional mode, hence in Table 4 , it can be observed that torsional mode 4 is unstable for cases 1, 2 and 3. Table 4 compares the torsional mode eigenvalues for the three cases. It is observed here that the increase in damper windings increases the damping in Mode 0 significantly.

Table 4. Eigenvalues of the SMIB System for Case 1, 2 and 3 for a Fixed Value of Xc=0.174 pu

\begin{tabular}{cccc}
\hline & Case 1 & Case 2 & Case 3 \\
Mode & 1.1 model & 2.1 model & 2.2 model \\
\hline Mode 5 & \multicolumn{2}{c}{ Torsional modes } & \\
Mode 4 & $0.04818 \pm 203.62 \mathrm{i}$ & $-1.850 \pm 298.16 \mathrm{i}$ & $-1.850 \pm 298.16 \mathrm{i}$ \\
Mode 3 & $-0.6336 \pm 160.67 \mathrm{i}$ & $-0.634 \pm 160.68 \mathrm{i}$ & $0.49165 \pm 203.71 \mathrm{i}$ \\
Mode 2 & $-0.0724 \pm 126.99 \mathrm{i}$ & $-0.0729 \pm 126.99 \mathrm{i}$ & $-0.634 \pm 160.69 \mathrm{i}$ \\
Mode 1 & $-0.2103 \pm 99.202 \mathrm{i}$ & $-0.217 \pm 99.230 \mathrm{i}$ & $-0.217 \pm 99.231 \mathrm{i}$ \\
Mode 0 & $-0.4308 \pm 8.8657 \mathrm{i}$ & $-0.48729 \pm 8.9107 \mathrm{i}$ & $-0.4889 \pm 8.9168 \mathrm{i}$ \\
& \multicolumn{4}{c}{ Electrical modes } & \\
Super-synchronous & $-4.37547 \pm 542.96 \mathrm{i}$ & $-4.5555 \pm 544.69 \mathrm{i}$ & $-4.6365 \pm 545.91 \mathrm{i}$ \\
Sub synchronous & $-4.19123 \pm 210.08 \mathrm{i}$ & $-4.27326 \pm 208.22 \mathrm{i}$ & $-4.49363 \pm 207.02 \mathrm{i}$ \\
\hline
\end{tabular}

\subsection{Effect of Exciter, PSS and SSSC}

Case 4: In this case, the SMIB system is modelled with a 2.2 synchronous machine model with a fast acting excitation system. Matcont result for the same is as shown in Figure 9a. The limit point is not encountered within the compensation range. Figure $9 \mathrm{~b}$ indicates that the common mode is unstable all along. The number of Hopf points for a SMIB system with 2.2 synchronous machine model is the same both under constant excitation and with a fast-acting exciter. However, the nature of the Hopf points change as shown in Table 5. 


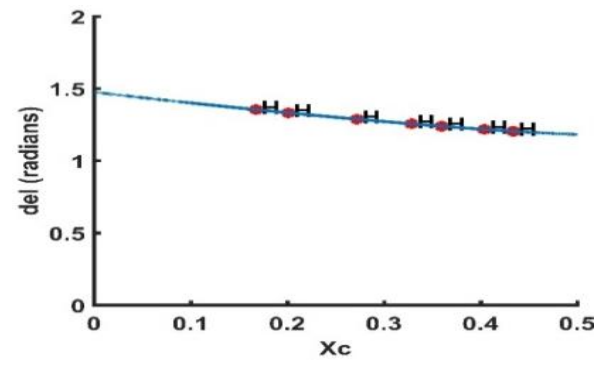

Figure 9a. Dynamic Bifurcations of SSR with 2.2 synchronous machine model equipped with a fast-acting exciter

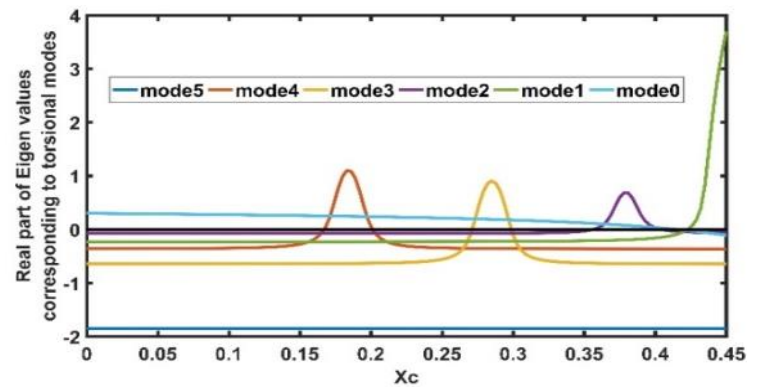

Figure $9 \mathrm{~b}$. Real part of eigenvalues corresponding to torsional modes v/s Xc for Case 4

Table 5. Xc values Corresponding to Hopf Points and Their First Lyapunov Coefficients for Case 4

\begin{tabular}{ccc}
\hline Xc pu & First Lyapunov Co-efficient & \\
\hline 0.167520 & $-1.700432 \mathrm{e}-05$ & Super-critical \\
0.201013 & $-2.506675 \mathrm{e}-05$ & Super-critical \\
0.271903 & $-1.426124 \mathrm{e}-04$ & Super-critical \\
0.328411 & $-6.816923 \mathrm{e}-02$ & Super-critical \\
0.359449 & $7.578238 \mathrm{e}-07$ & Sub-critical \\
0.403875 & $-3.459197 \mathrm{e}-05$ & Super-critical \\
0.433738 & $4.697554 \mathrm{e}-04$ & Sub-critical \\
\hline
\end{tabular}

Case 5: This case deals with bifurcation analysis of SMIB system modelled with a 2.2 synchronous machine model equipped with a fast acting excitation system and a PSS. The limit point is absent within the compensation region. Figure 10a and Figure 10b show seven Hopf points and it can also be noted that the addition of PSS to the system has stabilized mode 0 . Hence it can be said that the number and nature of the Hopf points for cases 4 and 5 remain the same

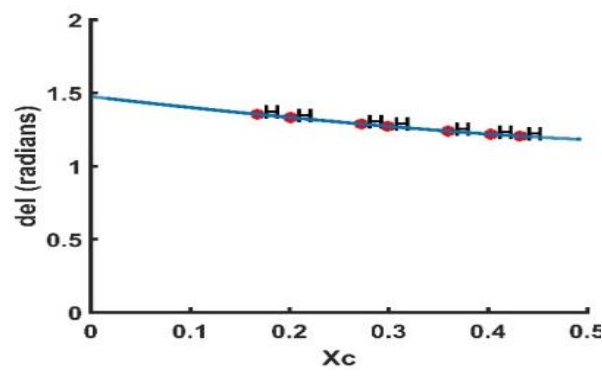

Figure 10a. Dynamic Bifurcations of SSR with 2.2 synchronous machine model equipped with a fast-acting exciter and PSS

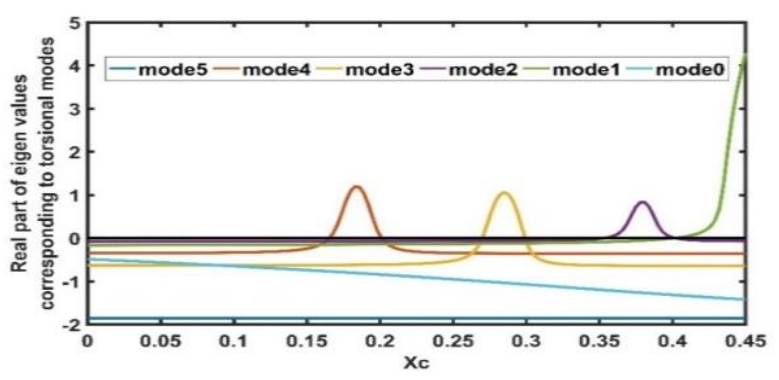

Figure 10b. Real part of eigenvalues corresponding to torsional modes v/s Xc for Case 5

Table 6. Xc Values Corresponding to Hopf Points and Their First Lyapunov Coefficients for Case 5

\begin{tabular}{ccc}
\hline $\mathrm{Xc} \mathrm{pu}$ & Lyapunov Co-efficient & \\
\hline 0.167711 & $-1.598730 \mathrm{e}-05$ & Super-critical \\
0.200876 & $-2.301412 \mathrm{e}-05$ & Super-critical \\
0.272180 & $-1.339217 \mathrm{e}-04$ & Super-critical \\
0.298817 & $-2.018532 \mathrm{e}-04$ & Super-critical \\
0.359797 & $8.214136 \mathrm{e}-06$ & Sub-critical \\
0.402832 & $-2.583189 \mathrm{e}-05$ & Super-critical \\
0.432231 & $2.064651 \mathrm{e}-04$ & Sub-critical \\
\hline
\end{tabular}

Case 6: This case deals with bifurcation analysis of SMIB system modelled with a 2.2 synchronous machine model equipped with a fast acting excitation system and a PSS. The series capacitor is replaced with 
a SSSC. The series compensation provided by SSSC is varied from $0 \%$ to $90 \%$ of the line reactance. Figure 11a and 11b show that the system does not yield any Hopf points, the system is sub-synchronous neutral.

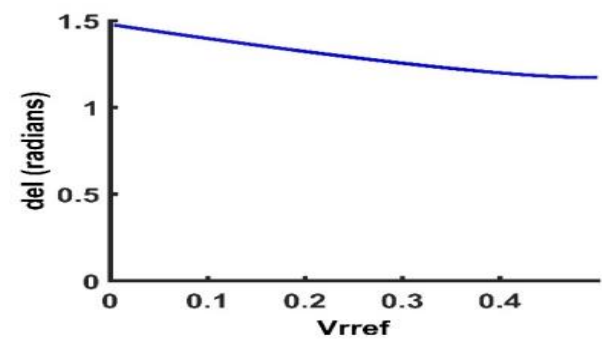

Figure 11a. Dynamic Bifurcations of SSR with 2.2 synchronous machine model equipped with a fastacting exciter, PSS and SSSC

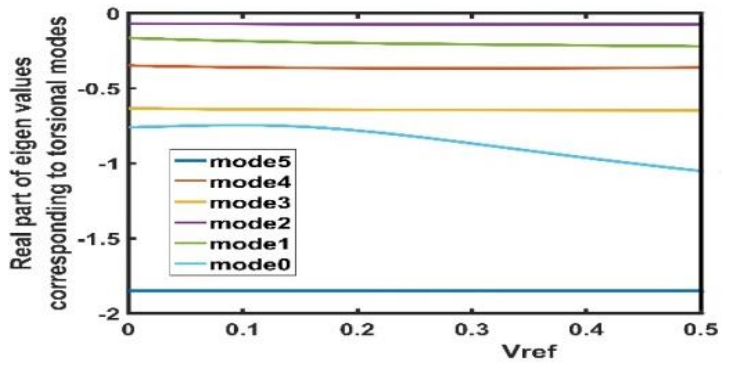

Figure 11b. Real part of eigenvalues corresponding to torsional modes v/s Xc for Case 6

Table 7 records the Eigenvalue corresponding to torsional and electrical modes for $\mathrm{Xc}=0.174 \mathrm{pu}$ for cases 3, 4, 5 and 6. Case 4 shows that eigenvalue for Mode 0 is unstable. On adding a PSS mode 0 gets stable. Replacing the fixed series capacitor makes the system Sub synchronous neutral.

Table 7. Eigenvalues of SMIB System for Case 4, 5 and 6 with Series Compensation=0.174 pu

\begin{tabular}{cllll}
\hline Mode & \multicolumn{1}{c}{ Case 3 } & \multicolumn{1}{c}{ Case 4} & \multicolumn{1}{c}{ Case 5 } & Case 6 \\
\hline Mode5 & $-1.8504 \pm 298.16 \mathrm{i}$ & $-1.8504 \pm 298.16 \mathrm{i}$ & $-1.8504 \pm 298.16 \mathrm{i}$ & $-1.8504 \pm 298.17 \mathrm{i}$ \\
Mode4 & $0.49165 \pm 203.71 \mathrm{i}$ & $0.48892 \pm 203.73 \mathrm{i}$ & $0.59148 \pm 203.74 \mathrm{i}$ & $-0.3370 \pm 202.97 \mathrm{i}$ \\
Mode3 & $-0.6341 \pm 160.69 \mathrm{i}$ & $-0.6364 \pm 160.69 \mathrm{i}$ & $-0.6207 \pm 160.69 \mathrm{i}$ & $-0.6284 \pm 160.60 \mathrm{i}$ \\
Mode2 & $-0.07294 \pm 126.99 \mathrm{i}$ & $-0.07368 \pm 126.99 \mathrm{i}$ & $-0.06836 \pm 126.99 \mathrm{i}$ & $-0.0686 \pm 126.98 \mathrm{i}$ \\
Mode1 & $-0.21738 \pm 99.231 \mathrm{i}$ & $-0.22859 \pm 99.227 \mathrm{i}$ & $-0.14664 \pm 99.267 \mathrm{i}$ & $-0.1519 \pm 99.099 \mathrm{i}$ \\
Mode0 & $-0.48890 \pm 8.9168 \mathrm{i}$ & $0.25025 \pm 9.5675 \mathrm{i}$ & $-0.78671 \pm 10.081 \mathrm{i}$ & $-0.598 \pm 8.8914 \mathrm{i}$ \\
& & Electrical modes & & --- \\
Super-synchronous & $-4.6365 \pm 545.91 \mathrm{i}$ & $-4.63062 \pm 545.92 \mathrm{i}$ & $-4.62432 \pm 545.93 \mathrm{i}$ & -- \\
Sub-synchronous & $-4.49363 \pm 207.02 \mathrm{i}$ & $-4.4170 \pm 206.99 \mathrm{i}$ & $-4.44517 \pm 206.97 \mathrm{i}$ & - \\
\hline
\end{tabular}

\subsection{Transient Analysis}

The transient response of the system for Case 3, 4, 5 and 6 is studied by introducing a sudden disturbance. This is done by reducing the mechanical torque by 0.1 p.u. for $0.1 \mathrm{sec}$. Series compensation of the system is fixed at $0.174 \mathrm{pu}$

Figure 12 shows that the SMIB system with constant excitation exhibits growth in oscillation in the rotor angle under sudden disturbances. Figure 13 shows the effect of a fast-acting excitation system. SMIB system incorporated with fast-acting exciter results in an unstable common mode. Modulation of the rotor angle can be observed in the transient response of the same.

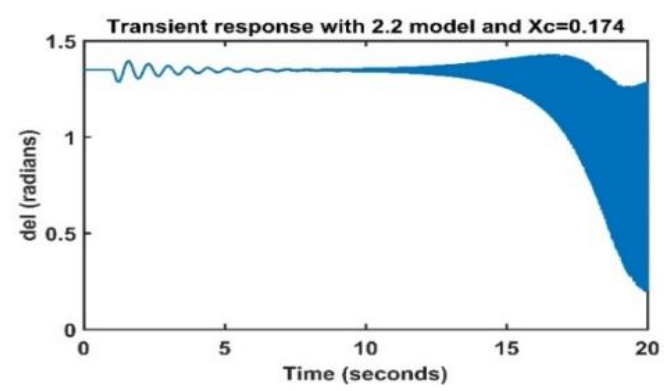

Figure 12. Transient response of SMIB under constant excitation

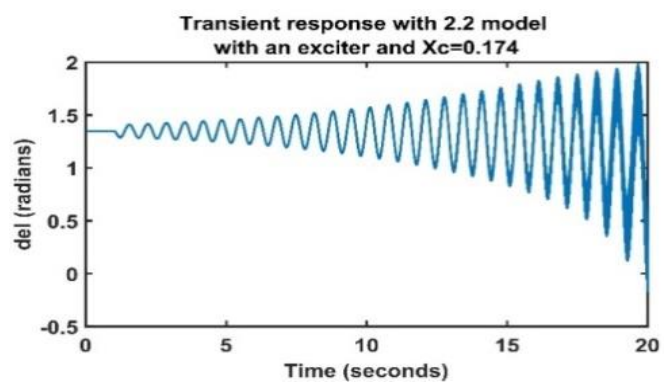

Figure 13. Transient response of SMIB with a fastacting exciter 
Figure 14 shows the effect of adding both, an exciter and a PSS. In both Figure 13 and Figure 14, the system exhibits growth in rotor angle under sudden disturbances. The system incorporated with SSSC shows an improved transient response as shown in Figure 15. The system becomes stable when the series capacitor is replaced by SSSC.

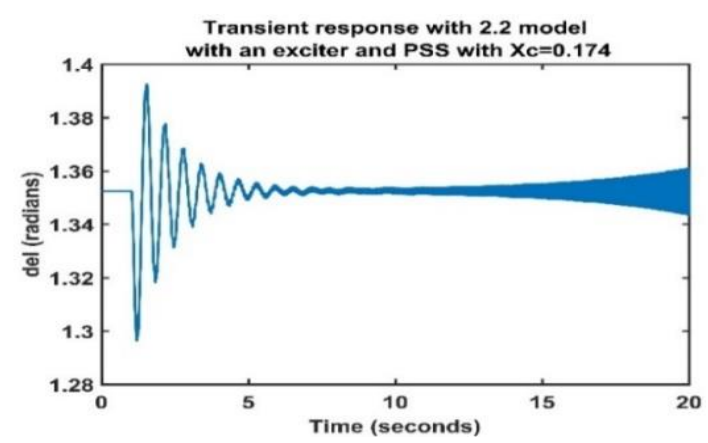

Figure 14. Transient response of SMIB with a fastacting exciter and PSS

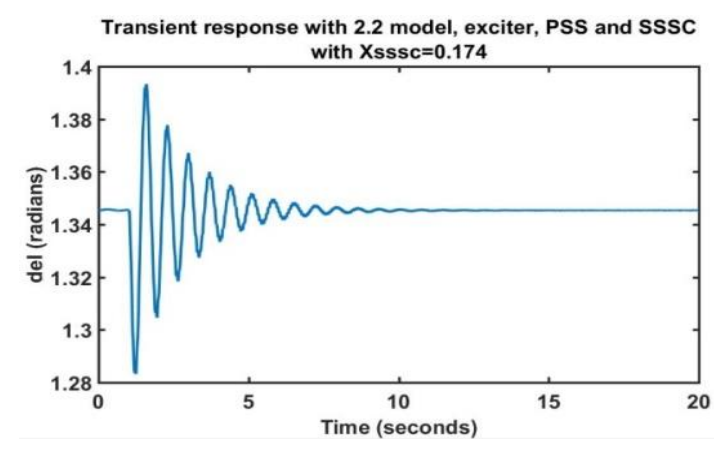

Figure 15. Transient response of SMIB equipped with a fast-acting exciter, PSS and SSSC

\section{CONCLUSION}

Bifurcation analysis carried out for the SMIB system under constant excitation led to the following conclusions and the same has been validated by Eigenvalue analysis and Transient analysis. Firstly, the increase in damper windings in the synchronous machine led to the occurrence of the first Hopf point for a lesser value of series compensation. Hence, it can be said that the stability boundary is more for a 1.1 synchronous machine model. It is also observed that the increase in damper windings increased the damping in torsional mode 0 significantly. Lastly, irrespective of the machine model adopted, the number and nature of Hopf points remain the same.

Bifurcation analysis of an SMIB model, when incorporated with a fast-acting exciter, caused mode 0 to go unstable. The absence of limit point bifurcation is observed both in the presence of a fast-acting exciter and PSS. Addition of a PSS along with the excitation system makes the mode 0 stable. Replacing the series capacitor with SSSC results in a stable system, as the system becomes SSR neutral and transient stability is achieved.

\section{APPENDIX}

The data for the SMIB system is taken from [19].

Exciter: $\mathrm{Ka}=200 ; \mathrm{Ta}=0.05 ; \mathrm{Tmd}=0.002 ;$ Efdmin $=-6$, Efdmax $=6$

PSS parameters: $\mathrm{Ks}=6 ; \mathrm{Tw}=10 ; \mathrm{T} 1=0.6 ; \mathrm{T} 2=0.5 ; \mathrm{T} 3=0.6 ; \mathrm{T} 4=1 ; \mathrm{Wn}=22 ; \mathrm{Vsmin}=-0.5$, Vsmax $=0.5$

SSSC rating: $150 \mathrm{MVA} ; \mathrm{bc}=0.359 \mathrm{pu} ; \mathrm{Rp}=278.7 ; \mathrm{gc}=1 / \mathrm{Rp}$

\section{REFERENCES}

[1] IEEE committee report, "Reader's guide to Subsynchronous Resonance”, IEEE Transactions on Power Systems. Vol. 7, No. 1, pp.150-158, February 1992.

[2] M. A. Pai, D.P. Sen Gupta, K.R. Padiyar, "Small-signal Analysis of Power System", Narosa Publishing House, ISBN-978-81-7319-5945.

[3] J. Hale, H. Kocak, "Dynamics and Bifurcation", Springer-Verlag, ISBN 0-387-97141-6.

[4] A.H. Nayfeh, Balakumar Balachandran, "Applied Non-Linear Dynamics”, WILEY-VCH Verlag GmbH \& Co. KGaA, Weinheim, 2004, ISBN-13: 978-0-47 1-59348-5.

[5] Rudiger Seydel, "Practical Bifurcation and Stability Analysis", Springer New York, Third Edition ISBN 978-14419-1739-3.

[6] J. W. Butler, C. Concordia, “Analysis of Series Capacitor Application Problems”, AIEE Transactions. Vol. 56, (1937), pp 975-988.

[7] D. N. Walker, D. A. Hodges, "Results of Sub-Synchronous Resonance Test Mohave", IEEE Transactions on Power Apparatus and Systems, Vol. PAS-94, No.5, September/October 1975.

[8] W. Zhu, "Analysis of Sub-Synchronous Resonance in Power Systems", PhD Thesis, Oregon State University, June 1994. 
[9] W. Zhu, R. R. Mohler, et.al., "Hopf Bifurcations in a SMIB Power System with SSR”, IEEE Proceeding of the 1995 Summer Meeting, pp. 531-534, PWRS.

[10] Rachananjali K, Suman S, et.al., "Implementation of Phase Imbalance Scheme for Stabilizing Torsional Oscillations." International Journal of Electrical and Computer Engineering (IJECE) Vol. 4, No. 5, October 2014, pp. 697-702 ISSN: 2088-8708.

[11] Narendra Kumar, Sanjiv Kumar, "SSR Alleviation using BVLC Supplementary Controlled SVS of Series Compensated Power System” TELKOMNIKA (Telecommunication Computing Electronics and Control) Indonesian Journal of Electrical Engineering Vol. 12, No. 9, September 2014, pp. 6551 - 6559.

[12] R.C. Mala, Nagesh Prabhu, H.V. Gururaja Rao, "Performance of STATCOM-ES in Mitigating SSR.", International Journal of Power Electronics and Drive System (IJPEDS) Vol. 8, No. 4, December 2017, pp. 1822-1829 ISSN: 2088-8694.

[13] R.C. Mala, Nagesh Prabhu, H.V. Gururaja Rao. "Hopf bifurcations of Subsynchronous resonance in a hybrid series compensated system with SSSC-ES", 2015 International Conference on Technological Advancements in Power and Energy (TAP Energy), 2015.

[14] A. M. Kulkarni "Power Systems Dynamics and Control", http://nptelonlinecources.iitm.ac.in

[15] Prabha Kundur, "Power System Stability and Control”, Tata McGraw-Hill, ISBN 13-9780070635159.

[16] K. R. Padiyar, "FACTS Controllers in Power Transmission and Distribution", New Age International Publishers, ISBN 978-81-224-2142-2.

[17] http://sourceforge.net/projects/matcont/files/matcont/matcont5p3/

[18] A. Dhooge, W.Govaerts, Kuznetsov, Meijer and Sautois, "New features of the software MATCONT for the bifurcation analysis of dynamical systems", Mathematical and Computer Modelling of Dynamical systems, vol. 14, no. 2, pp. 147-175, 2008.

[19] IEEE Subsynchronous Resonance Task Force of the Dynamic System Performance Working Group Power System Engineering Committee, "First Benchmark Model for Computer Simulation of Sub-synchronous Resonance", IEEE Transactions on Power Apparatus and Systems, Vol. PAS-96. No.5. September/October 1977.

\section{BIOGRAPHIES OF AUTHORS}

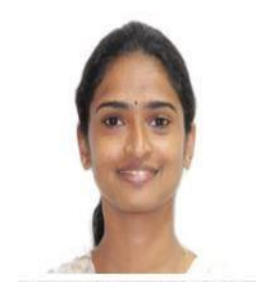

Ms Shruthi Ramachandra obtained her B.E in Electrical and Electronics from Visvesvaraya Technological University in 2010, M.Tech in Power Electronic Systems and Control from Manipal Institute of Technology in 2014. Currently, she is an Assistant Professor in Electrical and Electronics at Manipal Institute of Technology, Manipal. Her research interests include Power Electronics and Power Systems.

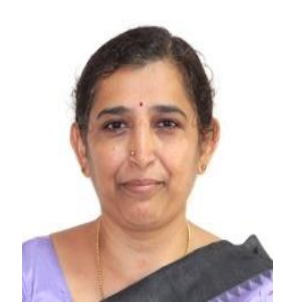

Mrs R C.Mala obtained her B.E in Electrical and Electronics Engineering from Mysore University in 1988, M.Tech in Power Systems from Mysore University in 1992. Currently, she is an Assistant Professor (selection grade) in Electrical and Electronics Engineering at Manipal Institute of Technology, Manipal and a research scholar at Visvesvaraya Technological University, Belagavi. Her research interests include Power system dynamics, Power electronics application to Power systems, Control systems. Mrs R.C.Mala is a Fellow of Institution of Engineers, India and member of Indian Society for Technical Education.

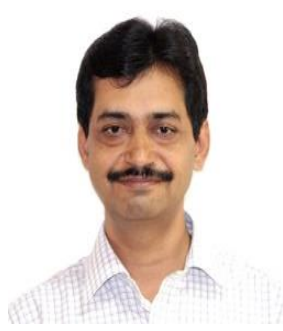

Mr H.V.Gururaja Rao obtained his B.E in Electrical Engineering from Karnataka University in 1989, M.Tech in Power Systems from Mysore University in 1993. Currently, he is an Assistant Professor (selection grade) in Electrical and Electronics Engineering at Manipal Institute of Technology, Manipal and a research scholar at Visvesvaraya Technological University, Belagavi. His research interests include Power system protection, Power electronics application to Power systems, Embedded systems. Mr H.V.Gururaja Rao is a Fellow of Institution of Engineers, India and member of Indian Society for Technical Education. 